\title{
Questioni di senso. Tra fenomenologia e letteratura
}

\section{Antonio Delogu, Donzelli, Roma 2017}

\author{
Recensione di \\ Roberta Guccinelli
}

Firmato da Antonio Delogu, già ordinario di Filosofia morale presso la Facoltà di Lettere e Filosofia dell'Università di Sassari, Questioni di senso. Tra fenomenologia e letteratura costituisce un utile strumento di lavoro per chiunque voglia (ri)avvicinarsi al mondo della vita o approfondirne la comprensione. Per chiunque, cioè, intenda prendere sul serio il mondo in questione, senza ignorare per questo la dimensione biofisica dell'organismo, nel rispettivo sistema di scambio con l'ambiente, o quella sociale dell'essere umano nel rispettivo contesto (la società). Diversi problemi attinenti a queste sfere esigono spiegazioni che possono dare le scienze naturali e quelle umane focalizzate sui comportamenti piuttosto che sulle condotte morali delle persone che esigono principalmente, anche se non esclusivamente, una comprensione di tipo intuitivo (intuizione eidetica) e una chiarificazione filosofica. Ci potremo orientare alla comprensione del mondo della vita e delle condotte delle persone che vi abitano, dopo aver modificato tuttavia l'atteggiamento, che fino a quel momento avevamo assunto (empirico o prefilosofico), in direzione di un vedere esemplare. Aver sottolineato e valorizzato conformemente all'intento del proprio lavoro una simile distinzione, di matrice fenomenologica, delle forme del sapere umano e delle ricerche e metodologie corrispondenti, è uno dei molti meriti di questo lavoro. Essa permette al lettore di muoversi più agevolmente, non solo nel mondo della vita, ma anche in quello della scienza, della quale si riconoscono ampiamente le virtù, nella Seconda Parte soprattutto del volume, mantenendo al tempo 
stesso una posizione critica rispetto a possibili bias scientistici. Gli equivalenti, nella sfera corrispondente, delle distorsioni pregiudiziali del senso comune. Questa prima mossa è di tipo epistemologico-metodologico.

La seconda mossa che Delogu compie in questo volume è di tipo etico. Ad essa veniamo immediatamente, non senza però aver prima suggerito la vastità degli interessi dell'Autore che in questo libro si intrecciano sapientemente. Delogu spazia dall'arte, alla psichiatria e psicopatologia, all'antropologia, alla Filosofia del Diritto. Si segnalano, dal punto di vista della Filosofia del Diritto, il capitolo sull'Originario senso del Diritto e le interessanti pagine, nella Seconda Parte del volume, sul fenomeno del banditismo barbaricino e sulla fenomenologia, correlativamente, del filosofo del diritto Antonio Pigliaru. Il lavoro che Pigliaru pubblica nel 1959, La vendetta barabaricina come ordinamento giuridico, rappresenta una buona esemplificazione, come mostra l'Autore, di fenomenologia applicata. Esso rivela, inoltre, la portata etica dell'esercizio fenomenologico, di cui il libro di Delogu offre tra l'altro ampia testimonianza; rivela, ancora, la capacità che lo stesso esercizio filosofico ha di allargare lo sguardo dell'“osservatore-agente” sulla realtà, invitandolo a riconoscerne la complessità, e l'eccedenza, rispetto a quanto possono catturarne le consuete griglie pratico-interpretative, permettendogli talvolta di modificare positivamente la stessa realtà, là dove le pessime condizioni di vita (sociali, comunitarie, economiche ecc.) lo richiedono. «Il filosofo orunese ha individuato» - scrive Delogu - «il potenziale di contestazione insito nell'inquieto pastore barbaricino alla conformità degli stili di vita che gli veniva imposta fin dalla adolescenza. Era fermamente convinto che la possibilità non la necessità è la cifra dell'umana esistenza per la quale la soggezione non è un destino mentre la liberazione è una scelta sempre possibile» ${ }^{1}$. Sulle idee politiche di Pigliaru non serve qua

\footnotetext{
${ }^{1}$ A. Delogu, Questioni di senso. Tra fenomenologie e letteratura, Donzelli Editore, Roma 2017, p. 232.
} 
pronunciarsi. È utile piuttosto, in questa sede, ricordare che il suo studio sul banditismo ha avuto un deciso impatto sulla vita e l'opinione democratica sarda.

Le considerazioni di Delogu sul saggio di Pigliaru ci consentono in ogni caso di illustrare più agevolmente la seconda mossa, da noi annunciata, che l'Autore compie appunto in questo lavoro: la mossa di natura etica.

Tornando al leitmotiv del volume di Delogu, quello del senso e della verità, possiamo aggiungere quanto segue. Chiunque abbia a cuore il mondo della vita, potrà tornare alle cose stesse, ricollocandosi sul rispettivo terreno originario, sul quale viene precisamente in luce la natura etica del fare fenomenologico, senza dover adottare una ragione estranea a quella incarnata esemplarmente dal fondatore della fenomenologia, Husserl. O da altri autorevoli fenomenologi (filosofi e psichiatri), come Merleau-Ponty, Scheler, Borgna, Callieri, Cargnello, spesso citati nel libro, assieme a grandi filosofe come Weil o Stein oppure De Monticelli. Chi intende "salvare" dunque il mondo della vita, restituendo ad esso, con l'attenzione che merita, i propri diritti ontologici, non dovrà ricorrere necessariamente a una razionalità funzionalistica o a un "Modello Classico di razionalità"2, nell'accezione searliana del termine, secondo il quale nella ragione (pratica) l'accento finisce per cadere sui mezzi; un modello che non lascia spazio, in quanto tale, a eventuali manifestazioni di gratuità. Si avverte invece, in questo libro, tutta l'importanza della gratuità del fare, e della gratitudine, vorremmo dire, che è possibile esprimere nei confronti della realtà, quando essa, almeno, si manifesta nei suoi aspetti degni (piuttosto che in quelli non degni): quando la fenomenologia, questa «filosofia a vocazione etica [...] consente di provare, per dirla con Simone Weil, "l'insondabile meraviglia del bene", liberandoci "dal dormiveglia delle chiacchiere" e, come direbbe Pessoa, "dalla sorditezza della

\footnotetext{
2 J. R., Searle, La razionalità dell'azione (2001), tr. it. di E. Carli e M. V. Bramè, Raffaello Cortina Editore, Milano 2003, pp. 4-30.
} 
quotidianità della vita"»33. In quell'istante, e solo in quell'istante - non possiamo evitare di aggiungere - noi trascendiamo noi stessi in direzione dell'eterno, direbbe Hersch.

Il volume costituisce un invito a "esplorare" i luoghi del mondo indicato, a farne esperienza in prima persona. Perché la ricerca di una «verità di senso» 4 o «verità incarnata nel vissuto» ${ }^{5}$ del rispettivo portatore, pro-teso verso il mondo, non può che partire da lì: dall'antepredicativo. Un punto di avvio, questo, che Delogu condivide non a caso con la tradizione fenomenologica, nel cui ambito, lo ripetiamo, si iscrive l'indagine sul senso che qui conduce. La realtà (ontologica o assiologica) del mondo della vita suscita tuttavia forti perplessità in certe forme di filosofie della cultura, o forme di scetticismo oppure di mero soggettivismo. Esse rappresentano di conseguenza dei bersagli polemici agli occhi di Delogu, che difende al contrario una versione dinamica (non aristotelico-tomista) di adaequatio rei et intellectus nella quale si offrono precisamente le verità del mondo della vita ${ }^{6}$, cui non intende affatto rinunciare. Verità o essenziali modalità di quel mondo, appunto, da scoprire in tutta la sua ricchezza, che finisce invece per essere negato dal nichilismo. Se simili verità non dovessero esistere, come ritengono i nichilisti o i postmoderni; se dovessero ridursi a un semplice flusso di sensazioni, come nell'empirismo; se dovessero infine soffocare, come nel «monismo parmenideo» di Severino che Delogu, in un confronto serrato col filosofo dell'Eternità incontrovertibile, legge come «un castello kafkiano, implacabile nella sua fissità, angosciosamente lontano dai problemi delle concrete, umane esistenze» 7 o come un racconto «borgesianamente fantastico»8; se ipotesi simili, o anche solo una di queste, dovessero verificarsi, allora verrebbe meno

\footnotetext{
${ }^{3}$ A. Delogu, Questioni di senso, cit., p. 241.

${ }^{4}$ Ivi, p. 162.

5 Ivi, p. 161.

${ }^{6}$ Cfr. ivi, p. 131.

7 Ivi, p. 55.

8 Ivi, p. 49.
} 
quanto di più importante possa esistere nella vita ed esistenza delle persone: «il senso valoriale delle cose che ci ri-guardano» 9 .

Il mondo della vita coincide nel contesto che abbiamo delineato con il regno husserliano delle evidenze originarie o con quel «paesaggio», in terminologia merleau-pontyana, «in cui [...] abbiamo imparato» - prima ancora di aver imparato la geografia - «che cos'è una foresta, un prato o un fiume» ${ }^{10}$. L’Autore ci accompagna, in questo viaggio (di ritorno) zu den Sachen selbst, al tempo stesso filosofico-letterario, come recita il sottotitolo, adottando un linguaggio accessibile anche a chi non abbia familiarità con la fenomenologia, lo stile di pensiero da lui privilegiato. Un linguaggio diretto, capace di restituire, nella loro concretezza, i diversi tipi di esperienze che possiamo fare nel "nostro" mondo. Non in quello della scienza, appunto, e nemmeno in quello naturalisticamente inteso. Nel mondo piuttosto che innanzitutto abitiamo, come abbiamo detto, nel quale incontriamo non solo altri esseri, umani e non umani, ma anche alberi, ad esempio, opere d'arte o semplici oggetti di uso quotidiano. Neppure gli oggetti più banali, tuttavia, mancano di suscitare stupore, o inquietudine (in negativo), quando se ne afferri - in un atteggiamento ad essi adeguato sul piano filosofico -, mettendo fuori gioco giudizi scontati e pregiudizi, il lato meno convenzionale o propriamente valoriale. Perché uno scolapasta, addirittura, o un qualunque altro utensile, fra agli oggetti più prosaici, possa essere colto come fenomeno, e non come lo scolapasta che usiamo nel mondo naturale, o pre-filosofico, occorre assumere allora l'atteggiamento dell'epochè. Occorre evitare, in altre parole, di oltrepassare i limiti di quanto è dato. Si tratta di sospendere il sapere, ad esempio, o le informazioni che possiamo avere riguardo allo scolapasta. Solo allora possiamo indossarlo come lo indossa Don Chisciotte, senza timore di apparire scomposti o folli.

\footnotetext{
9 Ivi, p. 273.

${ }_{10}$ M. Merleau-Ponty, Fenomenologia della percezione (1945), tr. it. di A. Bonomi, Bompiani, Milano 2003, p. 17.
} 
Uno dei grandi meriti di Questioni di senso. Tra fenomenologia e letteratura consiste, a nostro avviso, nel fatto che Delogu abbia tenuto presente anche Scheler, di cui ci fa piacere segnalare, in questo contesto, l'edizione italotedesca, da noi curata, del Formalismo nell'etica e l'etica materiale dei valori apparsa nel 2013 per i tipi di Bompiani.

Anche se qualche pagina del libro, che non manca affatto di coraggio, ci lascia forse in minima parte perplesse per il tono a tratti tranchant che l'Autore assume - ad esempio dove accenna al cosiddetto "conformismo dell'anticonformismo» ${ }^{11}$ - possiamo senz'altro concludere in questi termini. Si tratta di un lavoro che sa restituire perfettamente l'«impalpabile mistero» della nostra esistenza. Dell'esistenza stessa, addirittura, che permette sia a Pessoa, sia a Borges di fare «esperienza di metafisica inquietudine» ${ }^{12}$. La "rosa" di Borges, quel mistero lo incarna:

Voglio rendere grazie al divino / labirinto degli effetti e delle cause / per il mistero della rosa / prodiga di colore che non vede / per i fiumi segreti e immemorabili / che convergono in $\mathrm{me}^{13}$.

\footnotetext{
11 A. Delogu, Questioni di senso, cit., p. 241.

${ }^{12}$ Ivi, p. 35.

13 J. L. Borges, L'Altro, lo Stesso, Adelphi, Milano 2002, pp. 199, 201, citato in A. Delogu, Questioni di senso, cit., p. 36.
} 